\title{
Stylistique, analyse du discours littéraire
}

\author{
Dominique Maingueneau \\ Université Paris XII \\ Institut Universitaire de France
}

\section{Introduction}

Le découpage de cette section du Congrès, qui associe stylistique et linguistique de l'écrit, permet de grouper de manière originale des recherches qui participent de divers champs. Dans ces quelques pages, pour la commodité je vais restreindre mon propos aux seules relations entre sciences du langage et littérature, tout en étant bien conscient que la «stylistique» pas plus que «l'écrit» ne se laissent cantonner dans ce domaine. A l'intérieur de cet espace limité je vais opérer une nouvelle restriction en centrant mon propos sur les problématiques d'analyse du discours littéraire, qui me semblent aujourd'hui particulièrement fécondes dans un paysage théorique déjà ancien et qui, bon gré mal gré, sont amenées mettre en cause une certaine conception des relations entre «linguistique » et "littérature », voire la cartographie institutionnelle des études littéraires.

\section{Analyse du discours et approches stylistiques}

Ici se pose immédiatement une question de terminologie. On peut considérer que l'étiquette de « stylistique » recouvre l'ensemble des concepts et des méthodes qui impliquent à la fois les sciences du langage et la littérature. Dans ce cas, les problématiques d'analyse du discours littéraire en font indéniablement partie. Néanmoins, il est clair que cela ne correspond pas à l'usage plus circonscrit qui prévaut en France, où la stylistique tire une bonne partie de son identité de l'existence d'enseignements et d'épreuves spécifiques dans les universités et les concours de recrutement des enseignants du second degré.

Cette discipline s'est considérablement enrichie dans les trois dernières décennies. L'introduction massive de notions issues des théories de l'énonciation linguistique, de la linguistique textuelle et des courants pragmatiques a considérablement modifié la manière dont on peut concevoir les relations entre « linguistique » et « littérature ». Ces nouvelles problématiques ont contribué à développer une stylistique du texte littéraire beaucoup plus performante. Les avancées en matière de déictiques, de modalisation, de genres de discours, de marqueurs d'interaction orale, de processus argumentatifs, d'implicite, d'anaphore, etc. ont permis d'entrer de plain pied dans une œuvre, de l'appréhender à la fois comme processus énonciatif et comme totalité textuelle. Plus précisément, on peut distinguer deux orientations complémentaires : l'une qu'on pourrait dire « micro » (a), et l'autre qu'on pourrait dire « macro » (b) :

(a) Quand on réfléchit en termes d'énonciation, on a accès à des phénomènes linguistiques d'une grande finesse (modalités, discours rapporté, polyphonie, temporalité, détermination nominale, métaénonciation...) où se mêlent étroitement la référence au monde et l'inscription de l'énonciateur dans son propre discours. Or la littérature joue énormément de ces «détails» auxquels les commentaires traditionnels n'avaient pas les moyens d'accéder. On pourrait dire que le "grain » de l'objet texte a été changé, comme si l'on avait utilisé un microscope beaucoup plus puissant.

(b) La réflexion sur l'énonciation permet en outre de passer sans solution de continuité d'une linguistique de la phrase à une linguistique du discours. Des notions comme celles de genre et de scène d'énonciation jouent ici un rôle crucial puisqu'elles contraignent les agencements textuels en les rapportant à l'énonciation comme activité qui s'exerce dans le cadre d'institutions de parole.

On le voit, une telle évolution est plus qu'un simple enrichissement méthodologique : elle amène à rompre la relation ancillaire entre linguistique et littérature qui prévalait dans les approches stylistiques classiques. Désormais, quand elles abordent le discours littéraire, les sciences du langage font davantage 
qu'aider le commentateur à analyser les ressorts d'une subtile machinerie esthétique, elles peuvent dire quelque chose sur l'œuvre elle-même en tant que celle-ci participe d'un certain régime du discours, saisi dans sa double face, linguistique et institutionnelle. Démarche qui met en cause l'opposition immédiate entre un «intérieur » du texte qui serait passible d'une approche stylistique appuyée sur une linguistique de la phrase et quelques pans de la rhétorique, et un «extérieur» sur lequel les sciences du langage n'auraient aucune prise. En appréhendant les œuvres comme participant du «discours littéraire », on déplace l'axe d'intelligibilité : du texte vers un dispositif de parole où les conditions du dire traversent le dit et où le dit renvoie à ses propres conditions d'énonciation (le statut de l'écrivain associé à son mode de positionnement dans le champ littéraire, les rôles attachés aux genres, la relation au destinataire construite à travers l'œuvre, les supports matériels et les modes de circulation des énoncés...) (Maingueneau 1993, 2004).

Pour autant, les approches classiques ne sont pas disqualifiées. On peut en effet distinguer quatre modalités dans les approches «stylistiques » au sens large, c'est-à-dire qui s'appuient sur les sciences du langage :

1) Selon la première, l'étude précise de phénomènes linguistiques contribue à l'interprétation d'un passage ou d'un texte singuliers découpés dans une ouvre, de façon à les rapporter à leur créateur et à son positionnement esthétique; on retrouve ici la démarche stylistique traditionnelle.

2) Selon la seconde, l'analyse s'efforce de caractériser linguistiquement un ensemble discursif construit comme corpus : ensemble de textes relevant d'un auteur, d'un genre, d'un positionnement...Cette fois, il s'agit avant tout de modéliser une zones de régularité.

Ces deux premières approches s'en tiennent à une étude proprement textuelle, même si les phénomènes considérés se trouvent être d'ordre énonciatif ou pragmatique.

1) Dans la troisième approche, l'analyse stylistique des œuvres s'ouvre à des problématiques plus compréhensives, à un réseau d'articulations qui inscrit les textes dans un ordre de réalité distinct, où genre de discours et scène d'énonciation subvertissent la frontière texte/contexte. L'analyste prend alors systématiquement en compte la dimension institutionnelle de l'énonciation littéraire. Ici l'étude s'inscrit clairement dans une perspective d'analyse du discours.

2) La quatrième approche va encore plus loin dans ce sens. Cette fois, ce ne sont plus les oeuvres qui sont l'unique objet, mais le discours littéraire, appréhendé comme réseau de genres très divers, comme zone de l'interdiscours. Ce «discours littéraire » ne se réduit pas, en effet, à l'étude des «grandes œuvres » littéraires : l'étude des pratiques de construction des canons, les modalités de l'enseignement de la littérature, les critiques dans les journaux ou les commentaires de type universitaire deviennent des objets pertinents, des pratiques discursives qui participent du fait littéraire. La manière dont les textes sont produits, circulent, dont ils sont consommés, la manière dont l'école gère le patrimoine littéraire... ne peuvent pas être dissociés de ce qui est considéré comme étant «à l'intérieur» du texte. Spontanément, la plupart des spécialistes de littérature opposent deux formes de subjectivité : celle de l' «énonciateur » et celle de l'individu « réel », du créateur considéré hors du texte. On sait que Marcel Proust, dans son Contre Sainte-Beuve, a théorisé une distinction de ce type ${ }^{1}$, mais elle porte en elle-même les germes de sa propre contestation : l'instance même qui a écrit ce Contre Sainte-Beuve ne se laisse pas capter par cette opposition élémentaire entre un moi interne à la création et un moi social étranger à la création... Il faut bien introduire une troisième instance : l'écrivain, qui joue sa partie dans le champ littéraire.

On le voit, par rapport à la stylistique traditionnelle, on assiste aujourd'hui à un double déplacement : 1) les analyses sont déportées vers l'étude de l'activité énonciative, 2) même les études qui s'en tiennent à l'étude des textes ne peuvent ignorer que les fonctionnements qu'elles dégagent peuvent être intégrés dans des problématiques d'analyse du discours qui ne sont pas «extérieures » aux sciences du langage. En effet, alors que les approches classiques des textes littéraires, qu'elles soient d'ordre psychologique ou d'ordre sociologique, acceptent de se tenir « hors » du texte, dans l'espoir de trouver une «articulation » entre texte et contexte, l'analyse du discours questionne l'idée même d'un " hors du texte ». Ce faisant, 
elle est sous la menace de deux dangers, qu'on pourrait nommer «textualisme » et «sociologisme »: la réduction de son objet d'analyse au seul texte, ou à la seule situation de communication, sans prendre en compte l'activité discursive.

Poser que le discours littéraire relève des « discours constituants » (Maingueneau et Cossutta, 1995), par lesquels se disent les paroles ultimes d'une collectivité, est une thèse qui m'est plus personnelle. Elle implique que l'on peut mieux comprendre le fonctionnement du discours littéraire si on l'intègre dans cet ensemble plus vaste des discours constituants dont elle assume de manière spécifique les invariants, plutôt que de s'en tenir à une coupure élémentaire entre discours littéraire et non-littéraire. C'est là un détour qui peut sembler coûteux, mais qui accroît l'intelligibilité du fait littéraire. Cette intégration dans les discours constituants a également pour mérite de rendre moins «lourde » l'étude du discours littéraire, puisque bon nombre de ses propriétés sont partagées par d'autres types de discours. Ainsi l'appartenance à un champ où se délimitent des positionnements concurrents, l'élaboration de scènes d'énonciation et de code langagier à la mesure des contenus déployés, l'existence de communautés discursives...

\section{Un problème de frontières académiques}

On ne peut pas se cacher, néanmoins, que le développement, depuis les années 1990, d'une analyse du discours littéraire soulève des difficultés non seulement épistémologiques mais aussi institutionnelles. L'étiquette même d' «analyse du discours littéraire » implique que soit pleinement assumée l'unité du discours, dans toutes ses manifestations. Cette idée s'oppose en fait à des résistances qui viennent de deux bords, des littéraires traditionnels, bien sûr, mais aussi de la plupart des analystes du discours.

Beaucoup de spécialistes de littérature jugent tout à la fois illégitime et inefficient le recours à des problématiques d'analyse du discours. Ils ont en effet tendance à imposer une alternative redoutable : soit on traite les œuvres littéraires comme n'importe quel autre type de discours, et dans ce cas le terme «analyse du discours littéraire » constitue un oxymore, soit on reconnaît l'incommensurabilité de la littérature, et dès lors on disqualifie les démarches d'analyse du discours littéraire. Cette alternative repose sur un présupposé puissant, issu de l'esthétique romantique, selon lequel il faut séparer la littérature du reste des autres productions verbales d'une société : il y aurait d'une part les énoncés «transitifs», qui auraient leur finalité hors d'eux-mêmes, d'autre part les œuvres véritables, « intransitives », " autotéliques », qui ne pourraient être abordées qu'en partant du postulat de leur incommensurabilité. Ce présupposé contribue également à fonder une certaine distribution des tâches dans l'univers académique : la distinction entre les facultés de lettres, qui auraient en charge les œuvres véritables, et les facultés de sciences humaines et sociales, qui seraient naturellement vouées à l'étude des « discours sociaux » de second plan.

Si l'on accepte un tel présupposé, l'analyse du discours apparaît comme une entreprise à la fois inconséquente et illégitime qui ramène à l'ordinaire de la communication ce qui excède tout ordinaire et toute communication.

En fait, le problème n'est pas d'opposer les œuvres aux «discours sociaux », comme si les œuvres n'étaient pas d'une certaine façon des «discours sociaux», mais de prendre acte de la diversité des discours dans une société donnée. C'est précisément l'un des objectifs de l'analyse du discours que de rendre raison de cette diversité. Cela concerne d'ailleurs la littérature elle-même, qui varie dans le temps et l'espace : on sait que la notion même de « discours littéraire », considéré comme un ensemble compact, n'a véritablement de sens que depuis la fin du XVIII ${ }^{\circ}$ siècle.

Ces réticences de nombre de littéraires rejoignent paradoxalement celles des analystes du discours à l'égard de la littérature. Au moment où l'analyse du discours est apparue, dans les années soixante, en matière d'étude de textes il existait une répartition tacite du travail : les facultés de lettres analysaient les textes prestigieux, en prêtant une attention particulière à leur «style »; de leur côté, les départements de sciences humaines ou sociales avaient affaire à des textes de faible prestige, des «documents » qui n'étaient pas considérés comme passibles d'une approche stylistique et n'étaient étudiés que parce qu'ils donnaient accès à des réalités extralinguistiques. Le développement des approches d'analyse du discours 
a eu pour effet de convertir le regard sur ces «documents », qui ont bénéficié désormais d'une attention comparable à celle des textes étudiés dans les facultés de lettres, mais la répartition traditionnelle des tâches a perduré : les analystes du discours se sont habitués à ignorer les corpus de type littéraire, philosophique, religieux en tant qu'ils constituent des œuvres et pas seulement des exemplaires d'une routine discursive.

\section{Les deux paradigmes}

A partir du moment où l'analyste du discours reconnaît que la littérature relève des «discours constituants ", il est bien obligé d'accepter la nécessité d'un partage des études littéraires entre deux paradigmes, l'un relevant des sciences humaines et sociales, l'autre d'une herméneutique des œuvres. Dans le premier paradigme, le discours littéraire est pensé comme inscrit dans l'ensemble des pratiques discursives d'une société. Dans le second, le commentateur se donne avant tout pour mission de faire vivre le Thésaurus d'œuvres à travers lequel la communauté construit son identité.

Pour clarifier quelque peu la divergence entre ces deux «paradigmes», on détachera quatre points significatifs :

\begin{tabular}{|c|c|}
\hline 5 PARADIGME HERMENEUTIQUE & 6 PARADIGME DISCURSIF \\
\hline \multicolumn{2}{|l|}{ OBJET } \\
\hline Analyse restreinte aux Cuvres. & $\begin{array}{l}\text { Corpus défini en fonction de l'objectif de la } \\
\text { recherche ; il peut inclure des textes relevant de la } \\
\text { paralittérature, de genres connexes (commentaires, } \\
\text { manuels...) ou des textes relevant d'autres types de } \\
\text { discours. }\end{array}$ \\
\hline \multicolumn{2}{|l|}{ SINGULARITE } \\
\hline $\begin{array}{l}\text { Focalisation sur l'unicité de chaque œuvre et } \\
\text { celle de son commentateur. }\end{array}$ & $\begin{array}{l}\text { Focalisation sur les invariants du discours } \\
\text { littéraire, étude des conditions de possibilité de } \\
\text { l'unicité des œuvres. }\end{array}$ \\
\hline \multicolumn{2}{|l|}{ FINALITE } \\
\hline $\begin{array}{l}\text { Production de nouvelles interprétations, en } \\
\text { vertu du caractère inépuisable du sens des } \\
\text { œuvres. }\end{array}$ & $\begin{array}{l}\text { Étude des conditions de l'« interpretabilité » des } \\
\text { textes en un moment et un lieu donnés. }\end{array}$ \\
\hline \multicolumn{2}{|l|}{ COMMUNAUTES } \\
\hline $\begin{array}{l}\text { Spécialistes répartis pour l'essentiel en } \\
\text { communautés définie en fonction d'un intérêt } \\
\text { pour un auteur ou une période. }\end{array}$ & $\begin{array}{l}\text { Communautés de chercheurs partageant un certain } \\
\text { nombre de concepts et de méthodes. }\end{array}$ \\
\hline
\end{tabular}

Ce tableau ne doit pas susciter de malentendus : il ne s'agit pas de deux mondes étanches. En effet, les tenants des approches herméneutiques réinvestissent constamment les travaux du «paradigme discursif » en fonction de leurs finalités propres. De leur côté, les tenants du paradigme discursif ne peuvent pas travailler sans s'appuyer sur l'arrière-plan herméneutique sans lequel il n'y a pas pour eux d'objet à 
étudier. Ce qui importe avant tout, c'est de prendre la mesure de la divergence entre les deux attitudes, une divergence qui n'exclut pas des hybridations continuelles à telle ou telle étape de l'analyse ; beaucoup de littéraires ne cessent en effet d'élaborer des compromis entre les exigences des sciences humaines et sociales et une visée herméneutique.

De toute façon, ceux qui opèrent à l'intérieur du paradigme discursif ne peuvent ignorer qu'ils participent d'une entreprise inévitablement très minoritaire. Toute sophistiquées qu'elles soient, les approches de type herméneutique se situent en effet dans le prolongement du rapport spontané aux œuvres, où ces dernières sont évaluées en fonction du vécu singulier ou collectif de sujets pris dans les multiples trames de la vie sociale. Les approches discursives, en revanche, sont indubitablement ascétiques et heurtent constamment l'idéologie spontanée des usagers, y compris des usagers que sont aussi les analystes du discours amateurs de littérature.

Le développement de problématiques d'analyse du discours a pourtant des conséquences qui sont loin d'être négligeables dans le monde universitaire : il est désormais très difficile aux démarches herméneutiques d'agir en toute innocence, d'ignorer qu'il existe d'autres manières d'envisager le fait littéraire, lesquelles participent d'un mouvement comparable à ce qu'a pu être l'émergence de la philologie à la jointure des $\mathrm{XVIII}^{\circ}$ et $\mathrm{XIX}^{\circ}$ siècles. Or la philologie, c'était à la fois le corrélat d'une nouvelle approche du langage, la grammaire historique, et un phénomène dont la portée excédait largement les études littéraires. Il en va de même pour l'analyse du discours, qui s'appuie sur un ensemble de transformations dans les sciences du langage en prise sur l'évolution de l'ensemble des sciences humaines et sociales.

\section{Références}

Maingueneau D. (1993), Le contexte de l'œuvre littéraire, Paris, Dunod.

Maingueneau D. (2004), Le discours littéraire. Paratopie et scène d'énonciation, Paris, Armand Colin.

Maingueneau D., Cossutta F. (1995), «l'Analyse des discours constituants », Langages, 117, 112-125.

${ }^{1}$ En fait, Proust ne parle pas d' « énonciateur », notion impensable dans la conjoncture intellectuelle dans laquelle il était pris, mais de « moi profond » ou de « moi créateur », opposé au « moi social », dit aussi « moi superficiel ». 